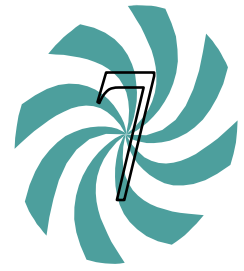

Tecnociencia, Vol. 22, $\mathrm{N}^{\circ}$ 1: 97- 108

enero-junio 2020

\title{
ENEMIGOS NATURALES DE Dione juno (Cramer, 1779) (LEPIDOPTERA: NYMPHALIDAE) EN CULTIVO DE Passiflora edulis Sims, J. (1818) CENTRO REGIONAL UNIVERSITARIO DE COCLE, UNIVERSIDAD DE PANAMÁ.
}

\footnotetext{
${ }^{1}$ Alonso Santos Murgas; ${ }^{2}$ Adnieska Martínez; ${ }^{3}$ Luis M. Rodríguez. ${ }^{1}$ Universidad de Panamá; Facultad de Ciencias Naturales Exactas y Tecnología, Departamento de Zoología. ${ }^{2,3}$ Centro Regional Universitario de Coclé, Estudiantes de la Universidad de Panamá, Facultad de Ciencias Naturales Exactas y Tecnología, Escuela de Biología. E-mail: Alonso.santos@up.ac.pa; santosmurgasa@gmail.com; adnieska2412@gmail.com, jimenezluis799@gmail.com

(D) orcid.org/0000-0001-9339-486X
}

\section{RESUMEN}

Muchas de las larvas de Lepidóptera producen daños a cultivos en sus primeros estadios del desarrollo, estas inhiben el crecimiento foliar de las plantas. Ya que las larvas en su crecimiento necesitan alimentarse constantemente en gran medida de hojas (plantas hospederas) para completar su ciclo biológico. En esta investigación se reportan los enemigos naturales de la mariposa Dione juno (Cramer, 1779) mediante un monitoreo de cuatro meses (agosto-noviembre 2019) en cultivos de Passiflora edulis Sims, J. (1818) sembrados en terrenos del Centro Regional de Coclé, Universidad de Panamá, sede de la provincia de Coclé. Mediante observaciones en Passiflora edulis, la planta hospedera natural de Dione juno, se identificaron en cada una de las etapas del desarrollo de su ciclo biológico los enemigos naturales; depredadores como hormigas: Hymenoptera, Formicidae, Solenopsis giminata (Fabricius 1804), chinche: Heteroptera, Pentatomidae, Mormidea notulata (Herrich-Schaeffer, 1844) y la avispas (Hymenoptera, Vespidae, Mischocyttarus angulatus Richards 1945) y 
parasitoides como, moscas: Diptera, Tachinidae, Lespesia archippivora (Riley). Consideramos que estos enemigos naturales son la causa de la disminución de la población de Dione juno en el cultivo de Passiflora edulis, en los cultivos de P. edulis, del Centro Regional Universitario de la Universidad de Panamá, en la Provincia de Coclé. El ciclo biológico de Dione juno dura aproximadamente \pm 30 días, desde la eclosión de los huevos hasta la etapa adulta.

\title{
PALABRAS CLAVES
}

Estadíos, Huevos, larva, pupas, depredadores, parasitoide, mariposa

NATURAL ENEMIES OF Dione juno (Cramer, 1779) (LEPIDOPTERA: NYMPHALIDAE) IN CULTURE OF Passiflora edulis Sims, J. (1818) REGIONAL CENTER UNIVERSITY OF COCLE, UNIVERSITY OF PANAMA.

\begin{abstract}
Many of the larvae of Lepidoptera cause crop damages in their early stages of development by inhibiting the foliar growth of plants; because the larvae need to feed constantly on leaves of the host plants to complete their biological cycle. This research reports the natural enemies of the Dione juno (Cramer, 1779) butterfly in crops of Passiflora edulis Sims, J. (1818) through a four-month monitoring (August-November 2019) in the lands of the University of Panama, Regional Center of Cocle province. Through observations in crops of Dione juno host plant (Passiflora edulis), natural enemies such as ants (Hymenoptera, Formicidae, Solenopsis giminata (Fabricius 1804); bugs (Heteroptera, Pentatomidae, Mormidea notulata (HerrichSchaeffer, 1844), wasps (Hymenoptera, Vespidae, Mischocyttarus angulatus Richards 1945), and parasites flies like (Diptera, Tachinidae, Lespesia archippivora) were identified in each of the development stages of its biological cycle. We consider these natural predators to be the cause of the decline in the population of Dione juno butterly, whose life cycle lasts about \pm 30 days, from hatching to adult stage in Passiflora edulis crops
\end{abstract}

\section{KEYWORDS}

Stages, eggs, larva, pupae, predators, parasitoid, butterfly. 


\section{INTRODUCCIÓN}

El orden Lepidóptera representa el segundo orden con más especies entre los insectos. Está compuesto por mariposas diurnas y polillas o mariposas nocturnas, cuya principal característica son, cuatro alas membranosas cubiertas por escamas. El ciclo de vida de los lepidópteros se compone de cuatro fases: huevo, larva (oruga), pupa (capullo o crisálida) y adulto. Cuando se encuentre en sus estadios larvario, su aparato bucal es de tipo masticador debido a que se alimenta de las hojas, flores, frutos, tallos y raíces de las plantas. Cuando es adulto, su aparato bucal se transforma en una espiritrompa y se alimenta de gran variedad de sustancias: néctar, fruta podrida, carroña, estiércol, orina y otros exudados vegetales y animales. Las mariposas poseen una fuerte relación con las plantas que les proporcionan alimento y refugio. Además, son muy sensibles a los cambios de temperatura, humedad y radiación solar que se producen por disturbios en su hábitat, debido a cambios en la vegetación y la cobertura de árboles (Luis-Martínez, 2003; Luis-Martínez et. al., 2000).

Los lepidópteros son considerados verdaderas plagas en cultivos extensivos, así como también en plantaciones comerciales de frutales y forestales. En los granos almacenados encontramos polillas cuyas larvas producen graves deterioros. Urretabizkaya, N. et. al., 2010.

La especie Dione juno es una mariposa que se encuentra específicamente dentro de la familia Nymphalidae. El género está compuesto de tres especies D. moneta (Hübner, 1825), D. glycera (C. \& R. Felder, 1861) y D. juno (Cramer, 1779). La distribución de $D$. juno va desde México hasta Paraguay (Hoskins, A. 2019). Esta especie, como las otras del género Dione, son de comportamiento migratorio, por lo que se les pueden encontrar en muchos hábitats y a altitudes entre a 0 metros a nivel de mar, hasta los 2000 metros, pero es más común en bosques perturbados entre 200-800 m. Por lo general, se encuentra en áreas soleadas a orillas de ríos, laderas de bosque, bordes de bosques y de carreteras, también son muy comunes en zonas urbanas visitando flores en los jardines.

Este trabajo tiene como objetivo identificar los enemigos naturales en campo de la especie de mariposa Dione juno en cultivos de Passiflora 
edulis ubicados en parcelas de los terrenos ubicados en el Centro Regional Universitario de Coclé, provincia de Coclé.

\section{MATERIALES Y MÉTODOS}

Se realizó un monitoreo dentro de los cultivos del Centro Regional Universitario de Coclé, ubicado en Llano Marín, Corregimiento del Coco, Distrito de Penonomé, Provincia de Coclé, Republica de Panamá, cuyas coordenadas son: N 8²9'14" W 80¹9'37” a 66 msnm, Fig. 1. Se realizaron observaciones biológicas en un lapso de 4 meses (agosto-noviembre 2019).

Con ayuda del GARMIN GPSmap 62s se registraron las coordenadas geográficas del sitio de muestreo. En una parcela del cultivo de Passiflora edulis, con un total de 35 plantas se muestreo y se registró las siguientes informaciones: presencia de huevos y estadios larvarios y adultos de la mariposa Dione juno, sobre las estructuras morfológicas de la planta Passiflora edulis.

Se observaron la presencia de parasitoides y depredadores. Se realizaron colectas de huevos para darle seguimiento diario controlado. Luego en el estadio larval 4 fueron llevadas a campo, debidamente marcadas para observar si estas eran parasitadas y luego recolectadas nuevamente, cuando alcanzaban el estadio de pre-pupa.

Se confeccionó una tabla de vida desde su ovoposición hasta alcanzar la etapa adulta. 


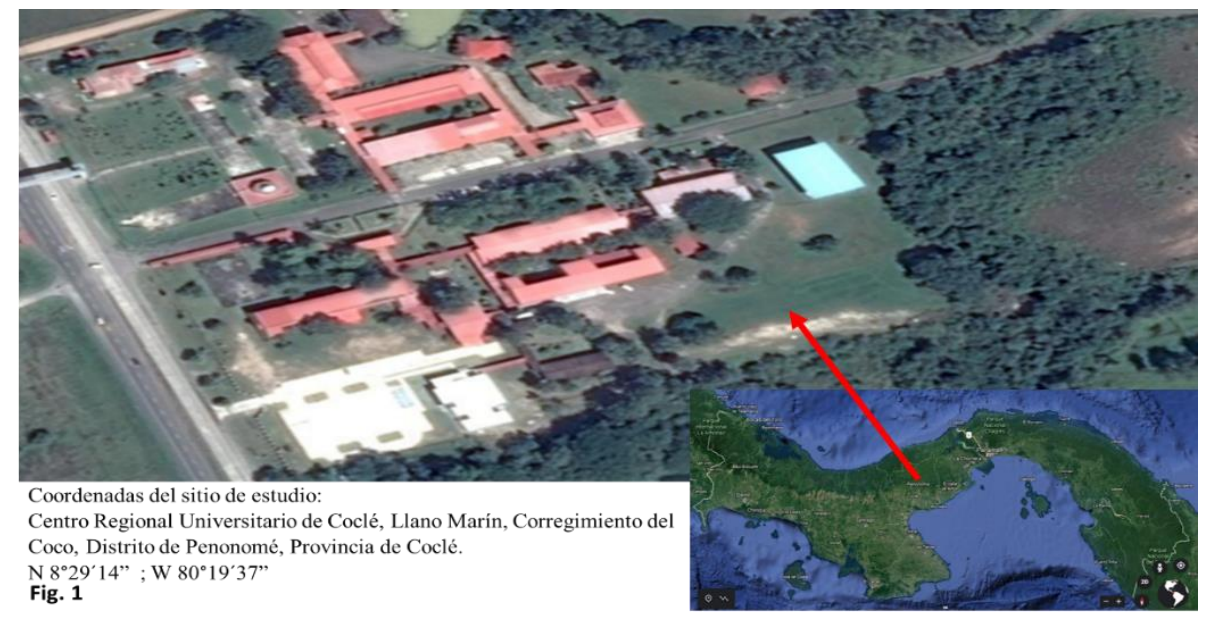

Fig. 1: Mapa y coordenadas del sitio de estudio.

Se estimó la supervivencia por cada estadio (huevo, larva, pupa), en el caso de la larva, se estimó el periodo que duró cada estadio larval (larva 1, 2, 3 y pre-pupa) Fig. 2. La tabla de vida se determinó a partir de huevos colectados el 3 de septiembre. Las larvas se alimentaron con hojas de Passiflora edulis. Se tomó en cuenta el número de huevos por oviposición (cuadro 1) y duración de cada estadio (cuadro 2).
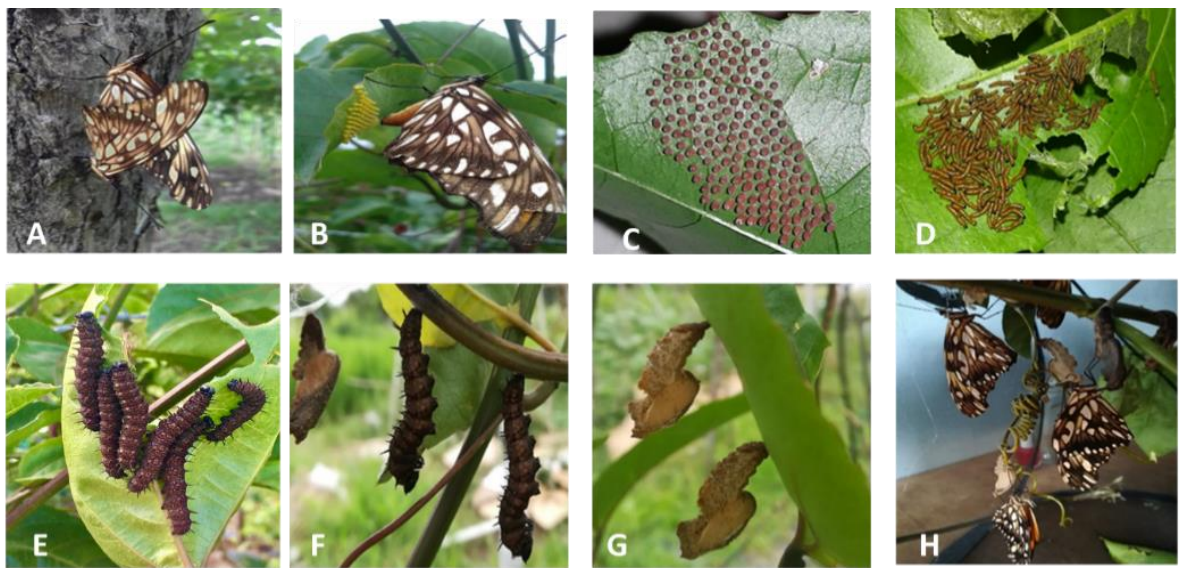

Fig. 2. Estadios de Dione juno. A. Cópula, hembra y macho; B. Hembra ovipositando sobre envés una hoja; C. Huevos; D. Segundo estadio larval; E. Tercer estadío larval; F. Pre-pupa; G. Pupa; H. Adulto de las mariposas emergiendo. 


\section{RESULTADOS}

Esta investigación se realizó en un periodo de 4 meses (agostonoviembre 2019), se hizo observaciones biológicas de Dione juno, en 35 individuos de $P$. edulis, planta hospedera de esta especie de mariposa, en una parcela del Centro Regional Universitario de Coclé.

D. juno copulan cerca de la planta hospedera (Fig. 2A) y ovipositan en el envés de las hojas de $P$. edulis aproximadamente entre 150 a 180 huevos en una sola puesta (Fig.2 B-C). Los huevos demoran en eclosionar aproximadamente 6 días, luego las larvas recién emergidas inician a alimentarse de las cascara (corion) de los huevos, este es su primer alimento. Luego pasa a ser herbívora, es decir a alimentarse raspando el parénquima de las hojas; en estos primeros estadios se alimenta constantemente desde las bases de las hojas hasta devorarlas en su totalidad.

Las larvas I Y II demoran aproximadamente 4 días cada una; la larva III, 3 días, en este estadio, la larva III se alimenta en mayor cantidad. (Fig.2E). La pre-pupa dura aproximadamente 2 días hasta llegar a la pupa (Fig.2 F y G). Las Larvas al momento de la pupación en la plata hospedera $P$. edulis, se ubica en las ramas, tallos y en el envés de las hojas, aunque también logramos observar pupas en troncos y estructuras cercanas a los cultivos como la cerca de alambres de la parcela. Las larvas pupan en grupos de 10 a 20. Las pupas tienen una duración de aproximadamente 8 días. Emerge preferiblemente en horas de la mañana. 

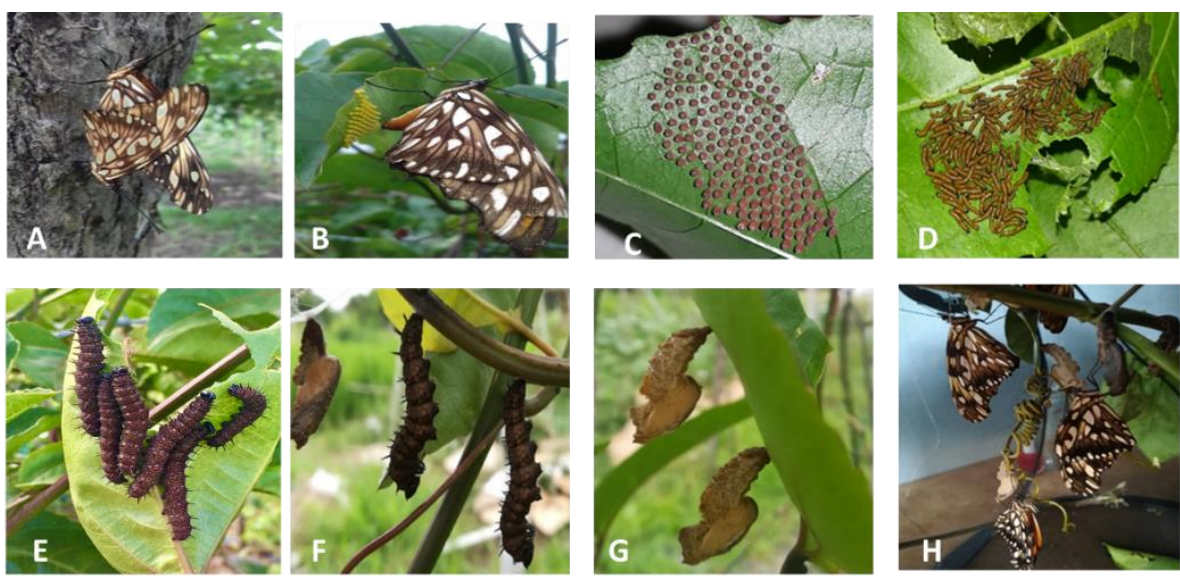

Fig. 2. Estadios de Dione juno. A. Cópula, hembra y macho; B. Hembra ovipositando sobre envés una hoja; C. Huevos; D. Segundo estadio larval; E. Tercer estadío larval; F. Pre-pupa; G. Pupa; H. Adulto de las mariposas emergiendo.

Cuadro 1. Tabla de vida de valores promedio para los estadios inmaduros de Dione juno.

\begin{tabular}{|l|c|c|c|c|c|}
\hline Estadio & $\mathbf{N}^{\circ}$. de Ind.(nx) & $\mathbf{l x}$ & $\mathbf{d x}$ & $\mathbf{\% d x}$ & $\mathbf{\% d o}$ \\
\hline Huevos & 80 & 0.00 & 11 & 13.75 & 13.75 \\
\hline Larva 1 & 69 & 0.86 & 19 & 27.54 & 23.75 \\
\hline Larva 2 & 50 & 0.72 & 5 & 10.00 & 6.25 \\
\hline Larva 3 & 45 & 0.90 & 4 & 8.89 & 5.00 \\
\hline larva 4 & 41 & 0.91 & 0 & 0.00 & 0.00 \\
\hline Pre-pupa & 41 & 1.00 & 6 & 14.63 & 7.50 \\
\hline Pupa & 35 & 0.85 & 22 & 62.86 & 27.50 \\
\hline Adulto & 13 & 0.37 & 0 & 0.00 & 0.00 \\
\hline
\end{tabular}

Nx. Número de individuos al inicio; lx. Proporción de individuos que sobreviven. Dx. Muertes; \% dx. Porcentaje de individuos muertos; \% do. Porcentaje de individuos muertos en la generación.

Los estadios más vulnerables dentro del ciclo biológico fueron: la larva I y II, ya que la mortalidad fue de $27.54 \%$ y $10.0 \%$, esta presenta una tasa baja de supervivencia ya que la competencia de alimentación fue Tecnociencia, Vol. (22), $N^{\circ} 1$ 
muy alta. En el caso de la pre-pupa y pupa, también se presentó una baja supervivencia debido a que suelen ser depredadas por avispas Mischocyttarus angulatus (Hymenoptera: Vespidae); hormigas, Solenopsis giminata (Hymenoptera: Formicidae) y chinches, Mormidea notulata (Heteroptera: Pentatomidae) y parasitada por moscas, Lespesia archippivora (Diptera: Tachinidae) (Fig. 3, Fig. 4, Fig. 5).

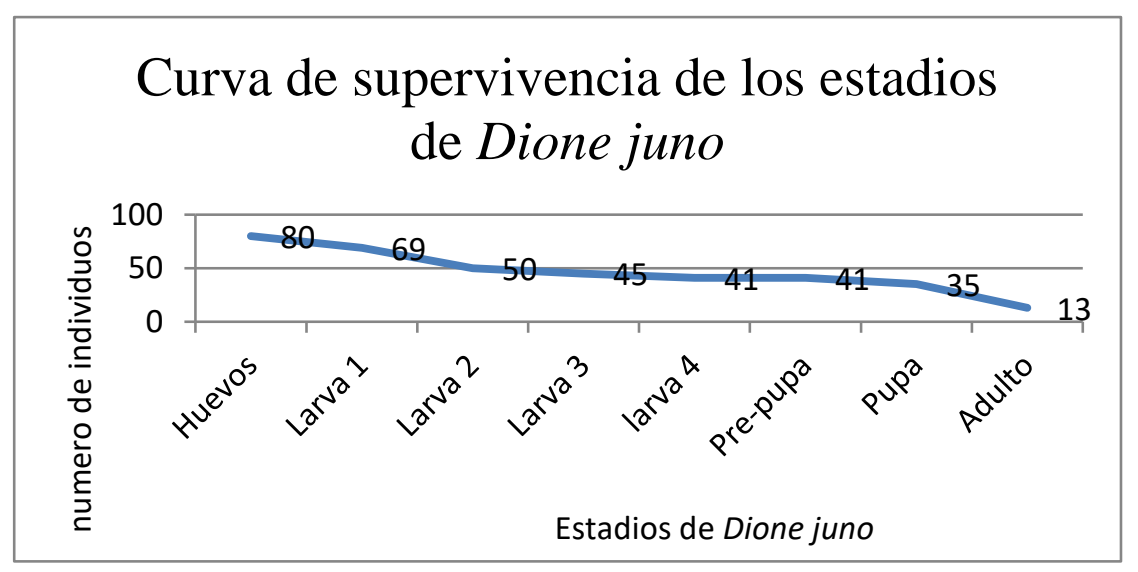

Fig.3. Curva de supervivencia de los estadios inmaduros de Dione juno. Esta muestra la tabla de vida.

Cuadro 2. Duración promedio en días de los distintos estadios inmaduros de Dione juno.

\begin{tabular}{|c|c|c|c|c|}
\hline Estadio & $\mathrm{N}^{\circ}$ de individuos & Dia & Duración & Supervivencia \\
\hline Huevos & 80 & 0 & 6 & 80 \\
\hline Larva 1 & 69 & 6 & 4 & 69 \\
\hline Larva 2 & 50 & 10 & 4 & 50 \\
\hline Larva 3 & 50 & 14 & 3 & 50 \\
\hline Larva 4 & 50 & 17 & 3 & 50 \\
\hline Pre-pupa & 45 & 20 & 2 & 45 \\
\hline Pupa & 35 & 22 & 8 & 35 \\
\hline Emergencia & 13 & 30 & $\Sigma=30$ & 13 \\
\hline
\end{tabular}


Este cuadro muestra el número de individuos que sobrevivían en cada uno de los estadios, además, el número de días que duro cada estadio. Los adultos emergieron en aproximadamente 30 días, en hora de la mañana (Cuadro 2). Las pupas emergieron aproximadamente entre 7-8 días.
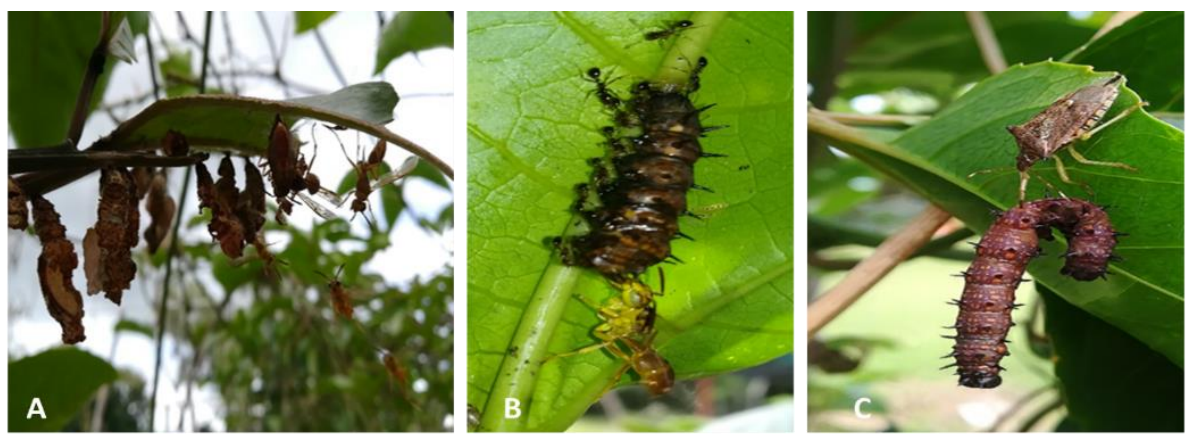

Fig.4. A. Se observa avispas Mischocyttarus angulatus depredando pupas de D. juno; B. Se observa avispas M. angulatus y hormigas Solenopsis giminata depredando una larva de $D$. juno. C. Se observa el chinche Mormidea notulata depredando una larva de $D$. juno.
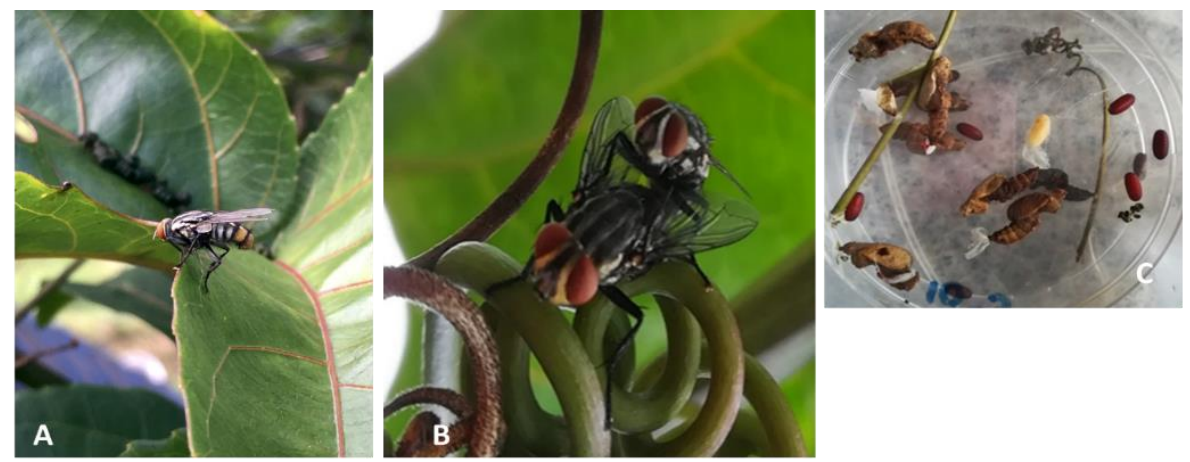

Fig.5. A. Hembra de Lespesia archippivora (Diptera: Tachinidae) sobre hoja de Passiflora edulis; B. Hembra y macho de Lespesia archippivora (Diptera: Tachinidae) copulando sobre zarcillos de Passiflora edulis. C. Larvas y Pupas de Lespesia archippivora (Diptera: Tachinidae) saliendo de las pupas D. juno.

\section{DISCUSIÓN}

La mariposa Dione juno tiene como hospedero natural a la Passiflora edulis, en cada uno de sus estadios larvarios hasta que emerge el adulto. Oviposita sus huevos en el envés de las hojas, coloca aproximadamente entre 150 a 180 huevos. En sus primeros estadios 
larvarios, se alimentan en gran magnitud, consumiendo casi en su totalidad las hojas y en ocasiones ramas de $P$. edulis, continúa alimentándose de las estructuras de la planta, hasta llegar a la prepupa; le toma casi 20 días hasta llegar a esta fase, al principio la larva presenta una tonalidad clara, incluso puede ser de color crema. Las larvas al momento de pupar se congregan, es muy probable que sea una estrategia defensiva contra depredadores y parasitoides.

Los controladores o enemigos naturales de $D$. juno, suelen presentarse con mayor proporción en los estadios III, pre-pupa y pupa. En el cual el principal depredador es la avispa Mischocyttarus angulatus, que suele devorársela completamente sin tener preferencia en pre-pupa o pupa, en estos dos estadios se presenta el mayor índice de mortandad. Luego los restos de las larvas y pupas son devorados por hormigas Solenopsis giminata, en menor cantidad.

Las moscas parasitoides Lespesia archippivora prefieren atacar a individuos de D. juno en los últimos estadios III y IV. Estas al entrar en la fase de pupa, la hace tornarse de un color café oscuro, esta característica es significativa ya que nos indica que la pupa está parasitada.

Es importante mencionar que la larva del parasitoide L. archippivora se desarrollan en una sola larva y luego pupa hospedera. El adulto del parasitoide L. archippivora coloca sobre el tegumento de la larva hospedera $D$. juno en el III u IV estadio un solo huevo, en aproximadamente 15-20 días antes de que ésta se transforme en pupa. Estos huevos pertenecen a la clase macrotipo en los cuales el embrión parasitoide no está completamente formado. La estrategia que utiliza la mosca parásita de colocar los huevos sobre el tegumento de su larva hospedera, le garantiza que el embrión complete su desarrollo y así pueda luego penetrar e invadir los tejidos de su hospedero para alimentarse de éste durante todo su ciclo larval. Esto a diferencia de los huevos microtipo en los cuales el embrión ya está completamente desarrollado (Calvo, 2004).

Las larvas parasitadas de $D$. juno continuaron alimentándose 
normalmente con la misma voracidad y no dieron muestras de estar parasitada. A los 22 días después de formada la pupa, ésta es de color café oscuro a negras, de contextura muy suave. Este aspecto es típico de las pupas enfermas de esta especie, cuando es atacada por microorganismos parásitos (Calvo, 2004 y 1992).

Esta estrategia en donde el parasitoide oviposita sobre la larva y sale posteriormente de la pupa, es uno de los mecanismos utilizados por los parasitoides koinobiontes endoparásitos para asegurarse una continua fuente alimenticia, ya que van consumiendo a su hospedero y le permiten continuar parcialmente con su desarrollo mientras el parásito se va desarrollando (Calvo, 2004; Gauld y Bolton 1988)

Estas investigaciones son importantes porque nos proporciona las identidades de los enemigos naturales que presenta $D$. juno, que se puede considerar una plaga sobre los cultivos de maracuyá (Passiflora edulis). De igual forma esta investigación nos indica cuales son los enemigos naturales en cada uno de los estadios de la especie.

\section{REFERENCIAS}

Calvo Renán, (2004). Parasitoidismo por dípteros en larvas de Caligo atreus (Lepidoptera:Nymphalidae)en Cartago, Costa Rica. Rev. Biol. Trop. 915-917.

Calvo,R. (1992). Observaciones sobre la Historia Natural de las larvas de Hylesia continua alinda (Druce) (Lepidoptera:Hemileucinae )en Coronado, Costa Rica. Brenesia 38:131-135.

Caldas Camacho, Danilo (2010). Artropodofauna En El Cultivo Tecnificado De "Maracuyá AMARILLO" (Passiflora edulis Var. 'Flavicarpa') Degener En El Distrito De Chinchao - Huanuco.

Gauld, I. \& B. Bolton. (1988). The Hymenoptera.British Museum of Natural History.Oxford University. Reino Unido.332 p.

Hoskins. A. Learn About Butterflies: la guía completa del mundo de las mariposas $\mathrm{y}$ las polillas, www.learnaboutbutterflies.com 
(Mariposas del amazona y los andes / Dione juno), último acceso [dic. 2019].

Sánchez-Jasso, Jessica M. Y Rivas-Manzano, Irma V. (2007). Ciclo Biológico Y Hábitos Alimentarios De Dione juno huascuma (Lepidoptera: Nymphalidae) Del Platanar, Malinalco, Estado De México.

Santos Murgas Alonso; Abrego J.C; Carranza, Raúl E.; Jaén, Luis A.; Szobotka, Roberto (2017). Enemigos Naturales De Estadios Inmaduros De Methona confusa Buther, 1873 (Lepidoptera: Nymphalidae) En Cerro Azul, Panamá.

Urretabizkaya, N.; Vasicek, A.; Saini, E. (2010). Insectos perjudiciales de importancia agropecuaria. Buenos Aires. ISBN: 978-987-1623-563.

Recibido 22 noviembre de 2019, 30 aceptado de diciembre 2019. 